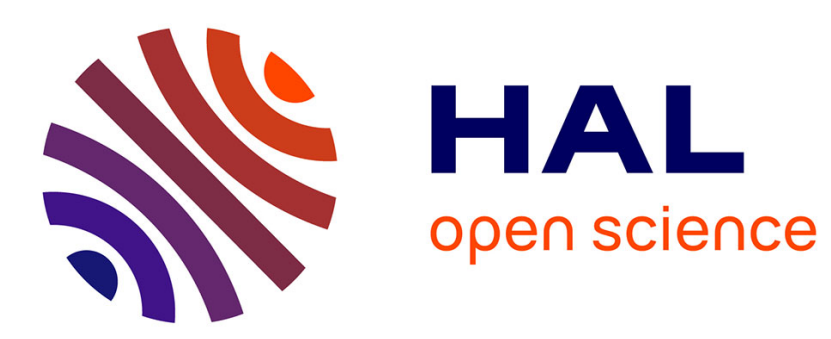

\title{
Influence of Tasks Duration Variability on Task-Based Runtime Schedulers
}

Olivier Beaumont, Lionel Eyraud-Dubois, Yihong Gao

\section{To cite this version:}

Olivier Beaumont, Lionel Eyraud-Dubois, Yihong Gao. Influence of Tasks Duration Variability on Task-Based Runtime Schedulers. [Research Report] INRIA. 2018. hal-01716489v2

\section{HAL Id: hal-01716489 \\ https://hal.inria.fr/hal-01716489v2}

Submitted on 28 Feb 2018

HAL is a multi-disciplinary open access archive for the deposit and dissemination of scientific research documents, whether they are published or not. The documents may come from teaching and research institutions in France or abroad, or from public or private research centers.
L'archive ouverte pluridisciplinaire HAL, est destinée au dépôt et à la diffusion de documents scientifiques de niveau recherche, publiés ou non, émanant des établissements d'enseignement et de recherche français ou étrangers, des laboratoires publics ou privés. 


\title{
Influence of Tasks Duration Variability on Task-Based Runtime Schedulers
}

\author{
Olivier Beaumont, Lionel Eyraud-Dubois, Yihong Gao \\ Inria Bordeaux Sud-Ouest \\ LaBRI, Université de Bordeaux \\ Email: firstname.lastname@inria.fr
}

February 28, 2018

\begin{abstract}
In the context of HPC platforms, individual nodes nowadays consist in heterogenous processing resources such as GPU units and multicores. Those resources share communication and storage resources, inducing complex co-scheduling effects, and making it hard to predict the exact duration of a task or of a communication. To cope with these issues, runtime dynamic schedulers such as StarPU have been developed. These systems base their decisions at runtime on the state of the platform and possibly on static priorities of tasks computed offline. In this paper, our goal is to quantify performance variability in the context of HPC heterogeneous nodes, by focusing on very regular dense linear algebra kernels. Then, we analyze the impact of this variability on a dynamic runtime scheduler such as StarPU, in order to analyze whether the strategies that have been designed in the context of MapReduce applications to cope with stragglers could be transferred to HPC systems, or if the dynamic nature of runtime schedulers is enough to cope with actual performance variations.
\end{abstract}

\section{Introduction}

In the context of HPC platforms, accelerators such as GPUs are more and more commonplace in processing nodes due to their massive computational power, usually beside multicores. This induces a dramatic increase in the number and variety of HPC nodes, due to the combination of available resources and the use of both multicores and accelerators. In this context, developing and maintaining optimized hand-tuned applications for all these architectures turns out to be extremely costly. Moreover, CPUs and GPUs of the same node share many resources (caches, buses,...) and exhibit complex memory access patterns (due to NUMA effects in particular). Therefore, it becomes extremely difficult to predict precisely the durations of both tasks and data transfers, on which 
traditional static offline schedulers rely to allocate tasks, even for very regular applications such as linear algebra kernels.

This situation favors dynamic scheduling strategies where decisions are made at runtime based on the (dynamic) state of the machine and on the (static) knowledge of the application. The state of the machine can be used to allocate a task close to its input data, whereas the knowledge of the application can be used to favor tasks that are close to the critical path. In the recent years, several such task-based runtime systems have been developed, such as StarPU [1], StarSs [2], or PaRSEC [3]. All these runtime systems model the application as a Directed Acyclic Graph (DAG), where nodes correspond to tasks and edges to dependencies between these tasks. These runtime systems are typically designed for linear algebra applications, and the task typically corresponds to a linear algebra kernel whose granularity is well suited for all types of resources. At runtime, the scheduler knows (i) the state of the different resources (ii) the set of tasks that are currently processed by all non-idle resources (iii) the set of ready (independent) tasks whose dependencies have all been resolved (iv) the location of all input data of all tasks (v) possibly an estimation of the duration of each task on each resource and of each communication between each pair of resources and (vi) possibly priorities associated to tasks that have been computed offline using heuristics like HEFT [4] or HeteroPrio [5,6].

In the context of MapReduce Applications running in datacenters, the variability of task running time is a well identified problem, for which several sophisticated strategies have been designed. They are in general based on the identification of slow tasks and their re-execution [7-9]. Re-execution is made easy by the use of distributed file systems based on replication such as HDFS [10]. The impact of stragglers and more generally of tasks that last longer than expected has been recently analyzed in [11] and our goal is to build the counterpart of this study in the context of HPC platforms. Indeed, this phenomena has been the object of very few studies in the context of dynamic schedulers on HPC platforms. It has however been observed and studied in the context of static schedulers. In $[12,13]$, it has been proved both in theory and practice that when the allocation and the schedule of tasks onto resources are computed in advance, the impact of unexpectedly long tasks is important. Dynamic runtime schedulers have been designed to cope with this variability and to overcome the limitations of static schedules, and are expected to perform much better in presence of tasks duration variability, since a long task will delay tasks that depend upon it, but not independent tasks statically allocated onto the same resource. Nevertheless, in runtime dynamic systems, the performance on a given kernel on a given resource is assumed to be constant [14], although it has already been noticed in [15] that some variability can be observed on task durations.

Our goals in this paper are (i) to study the variability of task durations using a dynamic scheduler like StarPU [1], on an typical HPC node consisting of GPUs and CPUs and (ii) to understand the impact of this variability on the makespan of an application. In our context and in order to deal with applications that consist in a large number of tasks belonging to few categories, and therefore to rely of significant statistics, we choose to concentrate on Cholesky 
and LU factorizations, for which very efficient implementations over StarPU are provided in the Chameleon library [16]. Due to lack of space, we only present Cholesky results in present paper and we refer the interested reader to [17]. Moreover, to understand the impact of task length variability on the makespan, we rely of the ability [14] to simulate accurately the execution on StarPU applications on top of Simgrid [18], a versatile simulator of distributed systems. The rest of this paper is organized as follows. We briefly survey related works and HPC background in Section 2. We analyze in detail tasks duration variability in Section 3 and Section 4, and its impact on the makespan in Section 5, before giving concluding remarks in Section 6 .

\section{Background}

Related Works: The problem of tasks duration variability has received significant attention in the context of distributed computing, first in Grid platforms [19] and later in Cloud computing platforms in the context of MapReduce environment [20]. Some of the work in this area consists one one hand in analyzing the variability of task execution times and trying to explain it $[21,22]$. On the other hand, several strategies have been designed to mitigate the effect of variability. Some works propose improved static scheduling strategies $[9,23,24]$ to find an assignment of tasks onto resources that minimizes the influence of variability. Others advocate the use of preventive task replication $[25,26]$ for some or all tasks of a job. Finally, studies have shown the interest of relying on duplication for tasks which last longer than expected [27, 28]. HPC platforms are thought of as being more predictable than Grid or Cloud platforms, and this problem has not been studied as much in HPC contexts. Stochastic task model where task durations are modeled with a (often Gaussian) distribution [12, 29], and the objective is to compute static schedules with minimal makespan and/or standard deviation [13]. Recent work [30] has analyzed the interest of two-phase robust scheduling, where the first phase is static and the second phase may react dynamically to actual execution times. However, to the best of our knowledge, there has been no attempt at characterizing the effect of the unpredictability of task execution times on the performance of dynamic schedulers as they are used in HPC runtime systems.

Runtime Systems: In this paper, we consider applications that use the STARPU [1] runtime system, and we execute them on a heterogeneous node, consisting of several CPU and GPU units. STARPU uses a Sequential Task Flow representation of applications, in which the computations are described as a set of tasks, with input and output data that describe dependences between tasks. The application developer provides different implementations for each task, so that they can be executed on a CPU or on a GPU. STARPU automatically infers dependencies between tasks based on which data they require, and uses this information both to schedule tasks on the appropriate processing unit, and to organize data transfers between the different memory units (main memory and 
memory of the GPUs). The execution times of tasks on the different resource types are monitored during execution, and the average execution time is used as a predictor for scheduling purposes. There is one such history-based model for the CPUs, and one for each of the GPUs. The default scheduling strategy in STARPU is called dmdas: it considers each task when all its dependencies have been satisfied, and assigns it to the processing unit that completes it the earliest (based on the current loads and estimated data transfer and execution times). Tasks assigned to the same processing unit are executed in priority order, where the priorities are provided by the application developer. Once a task has been assigned to a processing unit, but possibly before it starts executing if the resource is busy, the required data is prefetched to the memory of the processing unit if necessary. In the applications we consider, this allows to automatically overlap most of the communications with the computations.

\section{Task Durations}

Experimental Settings: In this section, we present some observations on the duration of tasks. We aim at providing a general experiment environment to study the variability of task durations on the different resources and the influence of this variability on the behavior of runtime schedulers. We consider a typical HPC node consisting of both multicore and accelerators. We made our experiments on the sirocco platform, which is made of 2 Dodeca-core Intel Xeon E5-2680, providing a total of 24 CPU cores, and 4 Nvidia GK110BGL GPU units. In STARPU, each GPU unit is managed by a dedicated CPU core, leaving 20 cores available for computation. In the rest of the experiments of this paper, GPUs are numbered from 0 to 3 and CPUs are numbered from 4 to 23 .

As mentioned in the introduction, in order to perform meaningful statistics, we rely on regular applications that involve a large number of tasks belonging to few categories only and for which efficient implementation within STARPU exist in the Chameleon library. The release versions that we use for experiments are StarPU 1.2.3 and Chameleon is 0.9.1. For both algorithms, we use the tiled implementation, which is obtained by dividing the input matrix in a certain number of tiles of fixed sizes, and applying the (Cholesky or LU) algorithm on tiles rather than on elements. Cholesky relies on 4 kernels, namely POTRF (Cholesky factorization) on the diagonal tiles, TRSM (triangular elimination), SYRK (symmetric matrix multiplication), and GEMM (matrix multiplication). For most experiments, we set the size of the matrix to $48000 \times 48000$ and the block size to 960 , what leads to $50 \times 50$ blocks matrices. The tile size is chosen so as to achieve good performance both on CPUs and GPUs. In order to compute statistics on the duration of factorizations, we perform both Cholesky and LU factorization 10 times each. In the case of Cholesky factorization, with $N=50$ blocks in each dimension, we therefore consider a total of 500 POTRF tasks $(N * 10), 12500$ SYRK and TRSM tasks $\left(\frac{N(N-1)}{2} * 10\right)$ and 196000 GEMM tasks $\left(\frac{N(N-1)(N-2)}{6} * 10\right)$, what is large enough to perform meaningful statistics of individual kernel durations. 


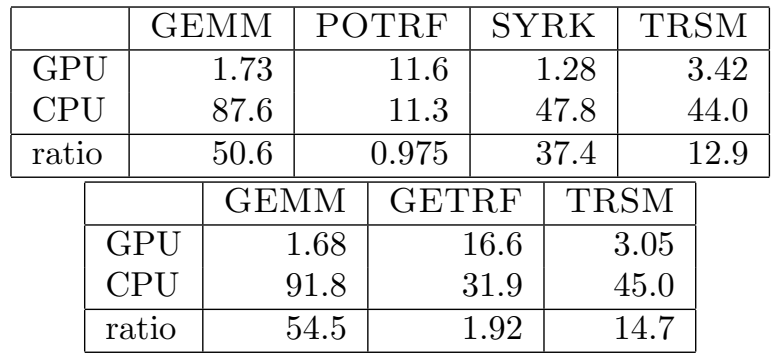

Table 1: Median task durations for Cholesky (top) and LU (bottom) factorizations, in ms.

Median Durations and Resource Allocation: The median values of tasks duration for each kernel of both LU and Cholesky factorization on each type of resource are given in ms in Table 1 . We can observe that, as expected, a GPU is in general much faster than a CPU core. On the other hand, we can also observe that resources are highly unrelated, in the sense that some kernels are highly accelerated on GPUs (up to 50 times for GEMM) whereas POTRF kernel is (slightly) slower on a GPU than on a CPU. In order to evaluate the overall computational power of the 2 Dodeca-core Intel Xeon E5-2680 and the 4 Nvidia GK110BGL GPU, we can estimate the time to perform all the kernels of a $50 \times 50$ Cholesky factorization without dependencies and without communication costs on 20 CPUs and 4 GPUs. We obtain 91.6s on the 20 CPUs and 10.1s on 4 GPUs, so that we can expect the 4 GPUs to perform about $90 \%$ of the work. In Table 2, we can observe that in a Cholesky run, STARPU allocates almost all GEMM tasks on GPUs, where they are highly accelerated, whereas it allocates almost all POTRF tasks on CPUs. Without dependencies, we can write a linear program (in rational numbers) to determine the optimal share between resources. This linear program proves that the processing time cannot be smaller than $8.25 \mathrm{~s}$. In the LP solution, CPUs perform all POTRF, SYRK and TRSM tasks, and GPUs perform $97.1 \%$ of GEMM tasks. Of course, due to dependencies, there may not be enough GEMM tasks to feed the GPUs, especially at the beginning and the end of the execution, what explains why GPUs also perform some POTRF, SYRK and GEMM tasks in an actual execution of the Cholesky factorization.

Tasks duration on CPUs: We focus on the case of CPUs, in particular in order to analyze NUMA (Non Uniform Memory Access) effects and the variability of task durations. Given the architecture of the 2 Dodeca-core Intel Xeon E5-2680, we split the CPUs into 4 groups, depending on their shared caches. In Figure 1, we depict the probability density function, i.e. the probability, for a kernel on a group to have for a given duration. We can observe several interesting phenomena on the GEMM plots. First, all groups exhibit a peak around $70 \mathrm{~ms}$, that corresponds to the processing time once data is in the cache. If data is not in the cache, then the necessary time to load it strongly depends 


\begin{tabular}{|l|r|r|r|r|}
\hline Cores & GEMM & POTRF & SYRK & TRSM \\
\hline GPU & 187533 & 48 & 3834 & 5861 \\
CPU & 8467 & 452 & 8416 & 6389 \\
\hline \% on GPU & $95.6 \%$ & $10.6 \%$ & $30.7 \%$ & $46.9 \%$ \\
\hline
\end{tabular}

Table 2: Task Sharing in Cholesky (top) and LU (bottom) factorizations.

on the group. In particular, for cores in group 3, we can observe a second peak around $175 \mathrm{~ms}$, that corresponds to the processing and data transfer durations. The same phenomenon, that will be denoted as NUMA effect in what follows, can also be observed on SYRK and TRSM kernels (we did not display the results for POTRF since we do not have enough tasks to perform this statistics). The second observation is related to duration variability. Indeed, even within the same group, we can observe that the time to process a given kernel onto a core varies from one execution to another. Note that the duration of a task is defined as the time between the end of the processing of this task and the end of the processing of the previous task allocated to the same resource. Note that for GPUs, STARPU uses data prefetching to overlap communications with computations, but not yet for CPU cores, that are seen as identical resources. Observed duration may include time spent to load the data. We expect that data prefetching and co-scheduling are responsible for task duration variability, but our focus in this paper is to observe variability and to measure its impact on the makespan, and we leave for future work the justification of observed phenomena. All these observations are very similar in the case of LU factorization, as shown on Figure 2.

Tasks duration on GPUs: Contrarily to the case of CPUs, we did not observe a significant variability between the 4 GPUs, so that we depict the aggregated statistics for all 4 GPUs. Figure 1 depicts the probability density functions for GEMM, SYRK and TRSM kernels in Cholesky factorization (we omit the results for POTRF for the same reason as before). We can observe a strong concentration of durations around the median value, showing that STARPU prefetching mechanism is efficient and that data transfers are in general well overlapped with processing. On the other hand, we can observe variability in task durations. The phenomenon is stronger for TRSM than SYRK than GEMM, but in all cases, we can observe that there are tasks whose duration is significantly larger than the median time. We will study in detail these long tasks in Section 4 and their impact on the overall processing time in Section 5.

The density functions for tasks in LU factorization are shown in Figure 4. The density of GEMM tasks behaves very similarly to the same kernel in Cholesky factorization, however the TRSM tasks show a very clear bimodal 


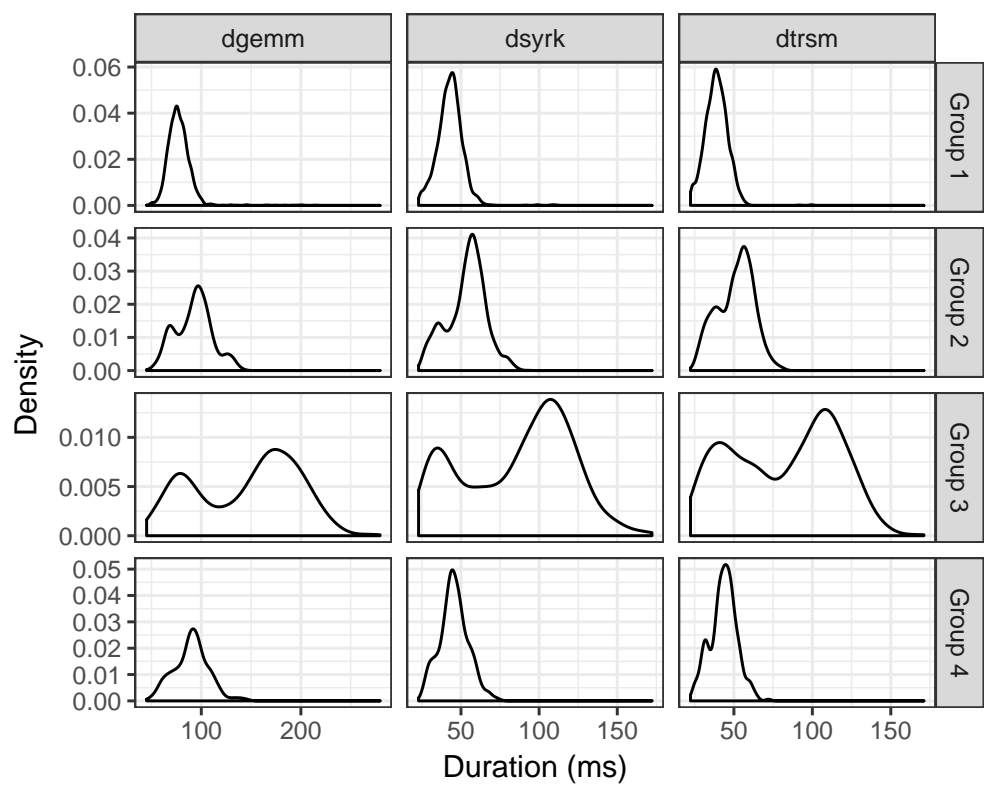

Figure 1: PDF of Cholesky task durations on CPU Cores

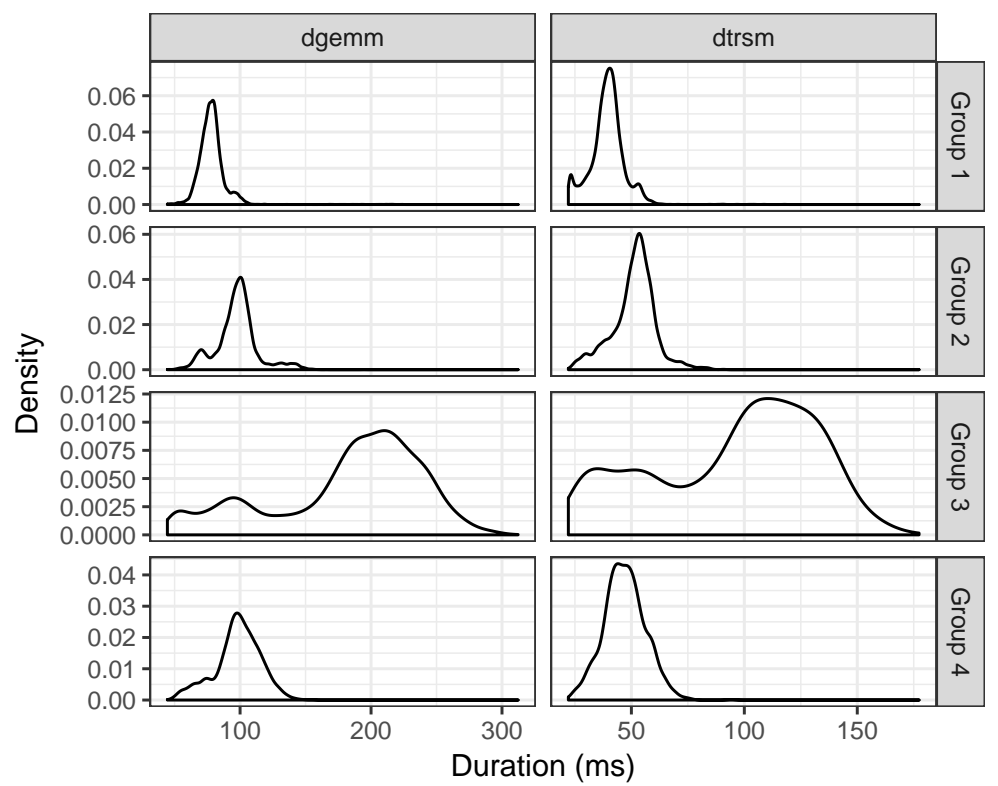

Figure 2: PDF of LU task durations on CPU Cores 

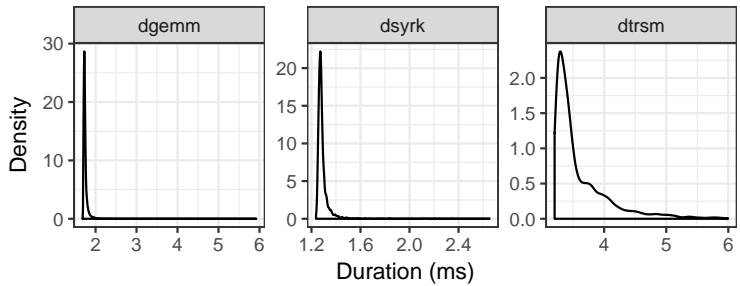

Figure 3: PDF of Cholesky task durations on GPUs

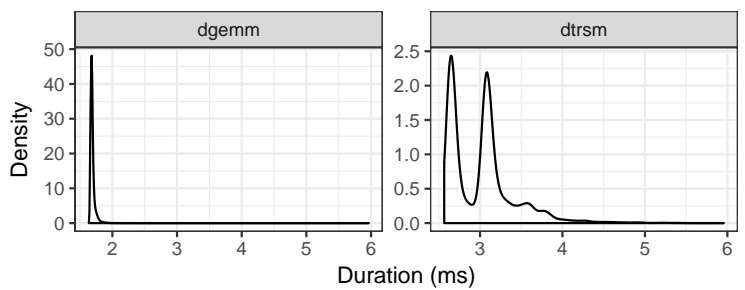

Figure 4: PDF of LU task durations on GPUs

distribution. This does not come from a behavior of the platform, but is rather a feature of the LU algorithm itself, which contains two different types of TRSM tasks (either on the L or on the U part of the solution). These tasks use different parameters of the TRSM kernel, and this results in sligthly different performance on the platform we consider. Technical limitations prevented us from considering both types of tasks separately.

\section{Long Delay Tasks Phenomenon}

In the context of MapReduce applications (see Section 2), it has been observed that beyond the intrinsic variability of task durations, there also exists tasks with abnormal duration, called stragglers. These tasks are critical and several works have been devoted to their early detection and to strategies to cope with them, based on re-execution, what is made possible by the use of distributed file systems based on replication such as HDFS. In the context of runtime systems such as STARPU, ad-hoc strategies would have to be added to the runtime system to cope with stragglers, what is out of the scope of this paper. Indeed, our goal is to identify if such phenomenon takes place in HPC systems and to quantify its impact on the application makespan, so as to evaluate the need for such solutions.

Detailed Statistics: The detailed distributions for task durations are shown in Table 4 for Cholesky factorization and in Table 4 for LU factorization. In addition, boxplots for each core and each GPU are depicted in Figures 4 and 4. 


\begin{tabular}{|l|r|r|r|r|}
\hline On GPU & GEMM & POTRF & SYRK & TRSM \\
\hline Minimum & 1.68 & 9.97 & 1.23 & 3.20 \\
\hline 1rst Quartile & 1.72 & 10.25 & 1.26 & 3.30 \\
\hline Median & 1.73 & 11.55 & 1.28 & 3.42 \\
\hline Mean & 1.75 & 23.98 & 1.29 & 3.63 \\
\hline 3rd Quartile & 1.75 & 17.84 & 1.29 & 3.76 \\
\hline Maximum & 29.20 & 175.1 & 2.65 & 29.66 \\
\hline \hline On CPU & GEMM & POTRF & SYRK & TRSM \\
\hline Minimum & 45.10 & 9.35 & 22.89 & 22.33 \\
\hline 1st Quartile & 74.33 & 9.59 & 40.05 & 36.96 \\
\hline Median & 87.60 & 11.27 & 47.76 & 44.02 \\
\hline Mean & 99.62 & 14.90 & 54.03 & 50.43 \\
\hline 3rd Quartile & 106.56 & 17.28 & 58.94 & 55.61 \\
\hline Maximum & 279.44 & 63.54 & 172.19 & 171.11 \\
\hline
\end{tabular}

Table 3: Statistics of Cholesky Task Duration (in ms) on GPU (top) and CPU (bottom).

In both Figures, we can clearly observe the CPU groups that we used in Section 3. Note that the fourth group is made of 2 cores only (instead of 6 for other groups) since STARPU dedicates 1 core per GPU to organize data transfers between the memory of the CPUs and the memory of each GPU, and the 4 cores have been chosen in this group. The boxplots show the minimum value, the first quartile, the median, the third quartile, the maximum value and the outlier values of tasks duration for each CPU core and GPU. The outliers can either be very slow tasks (negative outliers) or very fast tasks (positive outliers) in the experiments.

Outliers Identification: Based on the experiments depicted in Tables 4 and 4 and Figures 4 and 4, we can observe long delay tasks on both types of resources, but more outliers on GPUs. For instance, in the case of GEMM tasks for Cholesky factorization, on GPU, the largest processing time is almost 17 times larger than the median time, and on CPUs, it is "only" 3 times larger. If $Q_{1 s t}$ and $Q_{3 r d}$ denote respectively the value of the first quartile and the third quartile, outliers are classically identified in statistics with two threshold Outlier $_{+}$and Outlier - defined as Outlier $+=Q_{1 s t}-1.5 \times\left(Q_{3 r d}-Q_{1 s t}\right)$ and Outlier $_{-}=Q_{3 r d}+1.5 \times\left(Q_{3 r d}-Q_{1 s t}\right)$. Using this definition, we obtain the percentage of positive and negative outliers on both types of resources for all kernels, which are depicted in Tables 5 and 6 . We can observe that the number of positive outliers is always small, as expected, and that the fraction of negative outliers is much larger on GPUs than on CPUs. 


\begin{tabular}{|l|r|r|r|}
\hline On Gpu & GEMM & GETRF & TRSM \\
\hline Minimum & 1.64 & 15.76 & 2.57 \\
\hline 1st Quartile & 1.67 & 16.21 & 2.66 \\
\hline Median & 1.68 & 16.61 & 3.05 \\
\hline Mean & 1.69 & 17.28 & 3.02 \\
\hline 3rd Quartile & 1.70 & 17.73 & 3.15 \\
\hline Maximum & 13.60 & 23.64 & 7.41 \\
\hline \hline On CPU & GEMM & GETRF & TRSM \\
\hline Minimum & 44.5 & 18.7 & 22.1 \\
\hline 1st Quartile & 77.4 & 19.1 & 38.5 \\
\hline Median & 91.8 & 31.9 & 45.0 \\
\hline Mean & 106.3 & 41.2 & 51.5 \\
\hline 3rd Quartile & 107.1 & 45.9 & 54.6 \\
\hline Maximum & 312 & 278 & 177 \\
\hline
\end{tabular}

Table 4: Statistics of LU Task Duration (in ms) on GPU (top) and CPU (bottom).
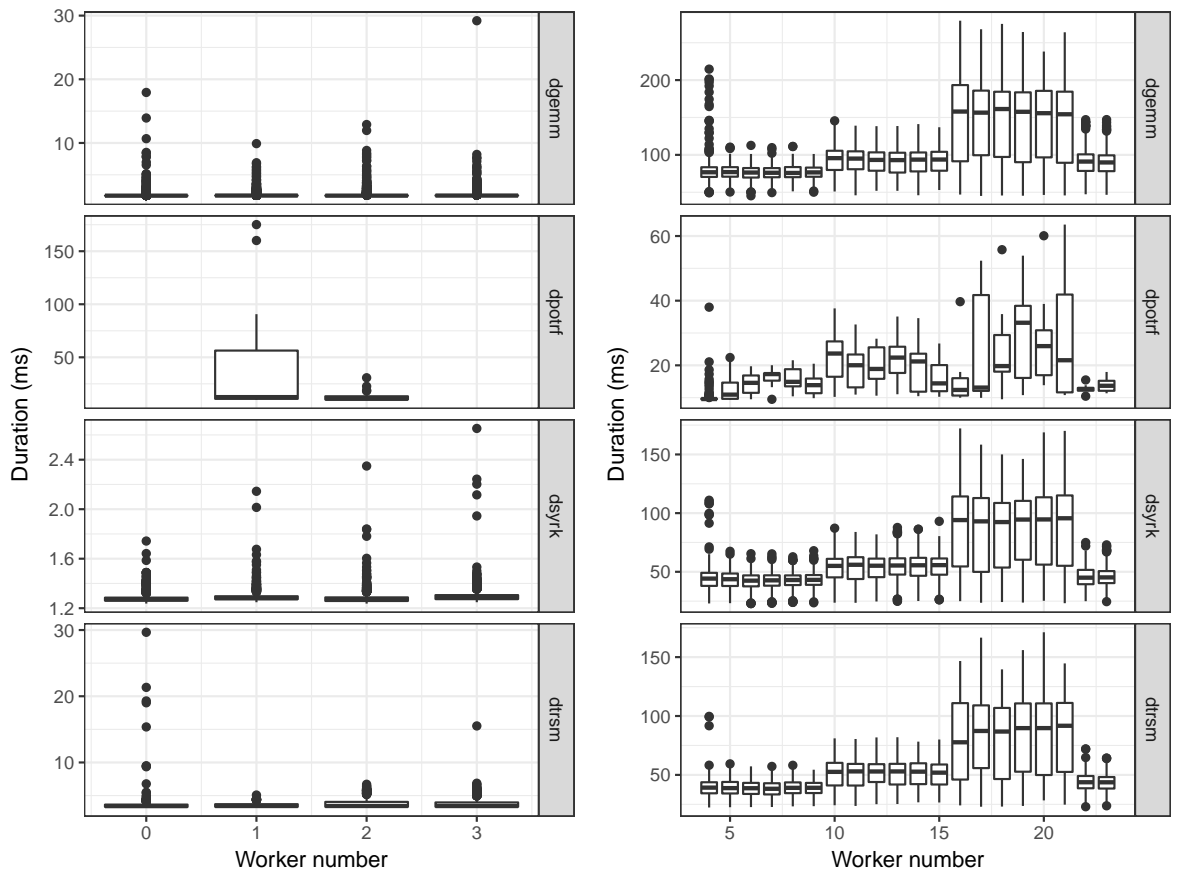

Figure 5: Task durations for Cholesky factorization for each worker on the GPUs (left) and the CPUs right) 

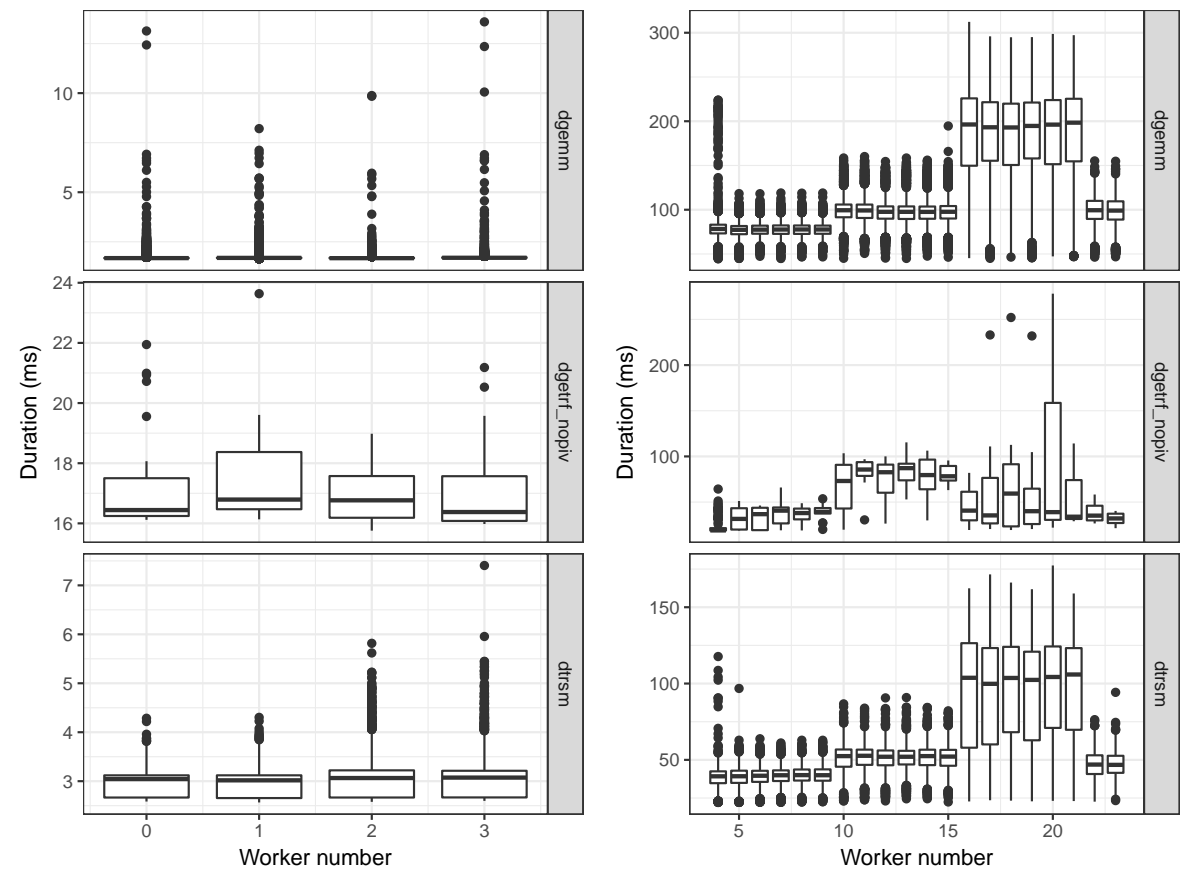

Figure 6: Task durations for LU factorization for each worker on the GPUs (left) and the CPUs right) 


\begin{tabular}{|c|c|c|c|c|c|}
\hline Type & GEMM & POTRF & SYRK & TRSM & total \\
\hline$C P U_{-}$ & $0.59 \%$ & $8.4 \%$ & $0.61 \%$ & $0.20 \%$ & $0.64 \%$ \\
\hline$C P U_{+}$ & $0.15 \%$ & $0.44 \%$ & $0.36 \%$ & $0.03 \%$ & $0.20 \%$ \\
\hline$G P U_{-}$ & $7.25 \%$ & $12.5 \%$ & $8.16 \%$ & $3.25 \%$ & $7.15 \%$ \\
\hline$G P U_{+}$ & $0 \%$ & $0 \%$ & $0 \%$ & $0 \%$ & $0 \%$ \\
\hline
\end{tabular}

Table 5: Percentage of Outliers in Cholesky factorization

\begin{tabular}{|c|c|c|c|c|}
\hline Type & GEMM & GETRF & TRSM & Total \\
\hline$C P U_{-}$ & $3.5 \%$ & $8.7 \%$ & $1.9 \%$ & $2.7 \%$ \\
\hline$C P U_{+}$ & $2.6 \%$ & $1.0 \%$ & $2.8 \%$ & $2.2 \%$ \\
\hline$G P U_{-}$ & $6.2 \%$ & $9.0 \%$ & $2.3 \%$ & $6.1 \%$ \\
\hline$G P U_{+}$ & $0.02 \%$ & 0 & 0 & $0.02 \%$ \\
\hline
\end{tabular}

Table 6: Percentage of Outliers in LU factorization.

\section{$5 \quad$ Influence of Negative Outliers}

The statistics presented in Section 3 and Section 4 are not sufficient to account for the influence of tasks duration variability on the makespan of applications. To do this, we rely on simulations to study the impact of both outliers and NUMA effects, by processing the same kernel with and without outliers and with and without NUMA effect. In both cases, the crucial point is that we do not keep the same schedule and the same allocation, what would induce a stronger impact of outliers, but we rather let the dynamic scheduling policy to make allocation decisions, thus enabling it to compensate the effect of an outlier by allocating less tasks later on this resource. To perform simulations, we rely on the implementation of StarPU over SimGrid [18]. This implementation provides an accurate estimation of the makespan of any STARPU application, including the runtime overhead and scheduling policy, over a simulated platform, taking into account time both for computation and for communication between the different memory nodes of the platform.

Methodology: The default behavior of STARPU is to record the execution times of each type of tasks during a real run of the application, and to use the average of measurements as input during the simulation: all tasks of the same class on the same type of resource have this exact duration, which implicitly takes into account cache effects since it is measured in an actual run in the normal condition for this application. We have augmented this behavior in two different ways. On the one hand, to account for heterogeneous workers, the duration of a task not only depends on its type, but also on the actual worker, which allows to simulate NUMA effects. On the other hand, to account variability of tasks duration, the duration of a task is not a constant value, but is randomly generated with a suitable distribution.

To obtain this distribution with a reasonable complexity, we have computed a 

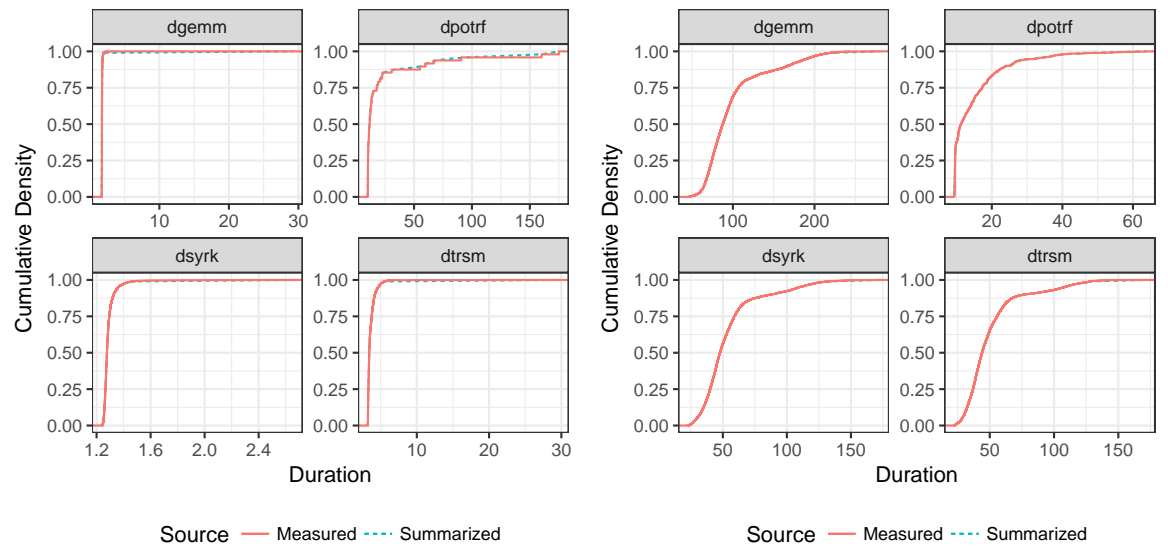

Figure 7: Comparison between the CDF and the piecewise linear approximation.

piecewise linear approximation of the empirical cumulative distribution function obtained from the measurements. This piecewise linear approximation uses 100 pieces, which appears both sufficient to provide a good approximation and small enough to allow fast random generation. These pieces are equally spaced along the $y$ axis of the CDF, so that the approximation remains correct even in the very dense parts of the distribution. The result for Cholesky application are depicted in Figure 7. It corresponds to the case of homogeneous workers, i.e. it contains measurements from all the GPUs on one side and from all CPUs and the other side. We can see that 100 pieces are enough to provide a very close approximation of the distribution. With this approximation, the data available to the simulator only contains the 100 breakpoints for each task type and for each worker. Each time a task is executed in the simulation, one interval is chosen at random, and then the duration is uniformy chosen at random between the two endpoints of the interval. Therefore, the repartition function of the distribution of the task lengths is exactly the piecewise linear approximation depicted in Figure 7.

Results: We performed the simulations for Cholesky factorization, with either large matrix size (48000, i.e. $N=50960 \times 960$ tiles) or medium matrix size (19200, i.e. $N=20$ ). In a first set of experiments presented in Figure 5, we study the variability of the overall makespan induced by long tasks and the influence of the heterogeneity of resources, mostly the NUMA effect of CPUs since GPUs achieve very close performance. In these experiments, as it would be done in practice, the dynamic scheduler of STARPU uses the same performance model for all CPU cores and a different performance model for each GPU. In the homogeneous case, the actual duration used in the simulation of a task on a CPU core does not depend on this specific core. In the heterogenous case, we use one distribution of tasks duration per core. In all figures, the red point 

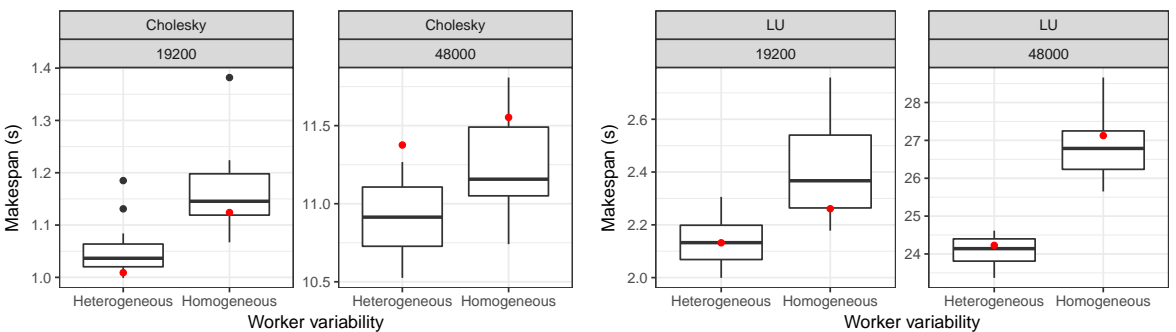

Figure 8: Sensitivity of makespan to tasks duration variability. Red dots show results without task variability.
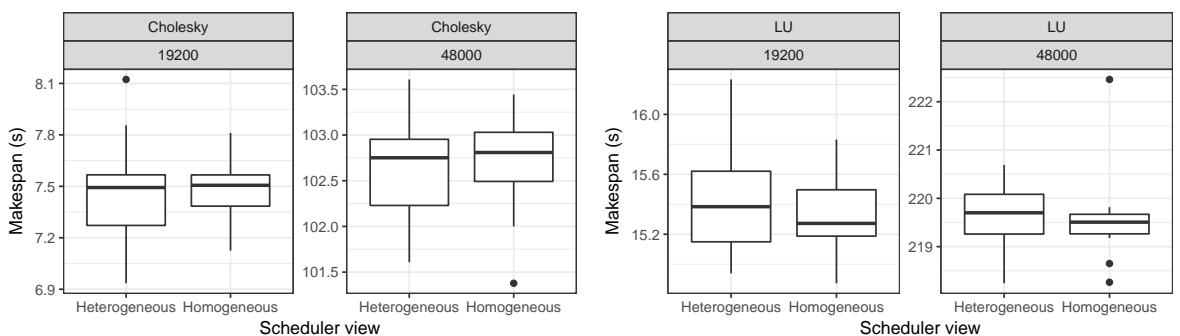

Figure 9: Sensitivity of makespan to different scheduler views of the platform.

corresponds to the case where the task duration on a CPU (resp. a GPU) is considered as constant and equal to the average value on CPU cores (resp. GPU units).

The first observation is that tasks duration variability actually induces a significant variability of the overall makespan. Indeed, even with the same exact distribution for task durations and simulations, the makespan varies by 6 to $10 \%$ from one execution to another, what is significant in a HPC context. The second observation is that heterogeneity helps! Indeed, even if the scheduler uses the exact same model for each CPU core, for both sizes, the heterogeneous makespan is always smaller than its homogeneous counterpart. This fact is not surprising since it is well known that having one resource with speed 2 is more efficient than two resources of speed 1. It proves that the dynamic scheduling strategy of STARPU is very efficient in taking heterogeneity into account by giving more tasks to the resources that request work more often, even if StarPU schedulers expect all CPU cores to be identical. The third observation is that task variability tends to degrade performance for smaller matrix sizes, whereas it tends to improve performance for larger sizes. We believe that this difference comes from the fact that dependencies between tasks are much more preponderant for smaller matrix sizes (since the width of the task graph is of the order of $N^{2}$, while its depth is of the order of $N$ ). The schedule is thus more sensitive to the presence of long tasks, since dependencies may prevent the scheduler from making corrections when a task lasts longer than 
expected.

In order to evaluate the ability of STARPU to cope with NUMA effect, we performed another set of simulations using CPU cores only. We depict in Figure 5 the makepan achieved by STARPU for both problem sizes and when the scheduler uses either the same performance model for all cores (Homogeneous) as done above or a specific performance model for each CPU core (Heterogeneous), what can be implemented in StarPU. We observe that StarPU dynamic scheduling strategy is in fact very robust to imprecisions in the performance model, and that considering an individual performance model does not improve makespan.

\section{Conclusion}

We consider the influence of the variability of tasks duration on the makespan of regular linear algebra kernels when using STARPU dynamic runtime system. Through direct experiments, we observe that due to complex co-scheduling effects in heterogeneous computing nodes consisting of muticores and accelerators, there is a significant variability in tasks duration. In particular, NUMA effect is clearly visible in multicore CPU nodes and there are stragglers tasks on GPUs. We design a sophisticated experimental settings based on simulations in order to study the influence of core heterogeneity on CPU nodes and stragglers on GPU nodes on the overall makespan. Our conclusion is that individual tasks duration variability actually induces variability in application makespan, but that the dynamic scheduling strategy of STARPU is very efficient in taking both tasks duration variability and resource heterogeneity into account, contrarily to what happens in presence of static scheduling strategies. This work opens many perspectives. On the practical side, it would be interesting to extend it to other types of nodes, other dynamic scheduling strategies and other applications. On the theoretical side, it would be interesting to explain achieved results and to better understand the stability of dynamic schedulers.

\section{References}

[1] C. Augonnet, S. Thibault, R. Namyst, and P.-A. Wacrenier, "Starpu: A unified platform for task scheduling on heterogeneous multicore architectures," Concurrency and Computation : Practice and Experience, vol. 23, no. 2, 2011.

[2] J. Planas, R. M. Badia, E. Ayguadé, and J. Labarta, "Hierarchical Taskbased Programming with StarSs," International Journal of High Performance Computing Applications (IJHPCA), vol. 23, no. 3, pp. 284-299, 2009. 
[3] G. Bosilca, A. Bouteiller, A. Danalis, M. Faverge, T. Hérault, and J. Dongarra, "PaRSEC: A programming paradigm exploiting heterogeneity for enhancing scalability," Computing in Science and Engineering, 2013.

[4] H. Topcuouglu, S. Hariri, and M.-y. Wu, "Performance-Effective and LowComplexity Task Scheduling for Heterogeneous Computing," IEEE Trans. Parallel Distrib. Syst., vol. 13, no. 3, pp. 260-274, Mar. 2002.

[5] O. Beaumont, L. Eyraud-Dubois, and S. Kumar, "Approximation proofs of a fast and efficient list scheduling algorithm for task-based runtime systems on multicores and gpus," in IPDPS, 2017.

[6] S. Kumar, "Scheduling of Dense Linear Algebra Kernels on Heterogeneous Resources," Ph.D. dissertation, Université de Bordeaux, 2017.

[7] M. Zaharia, A. Konwinski, A. D. Joseph, R. H. Katz, and I. Stoica, "Improving mapreduce performance in heterogeneous environments." in OSDI'08, 2008.

[8] S. Ibrahim, H. Jin, L. Lu, B. He, G. Antoniu, and S. Wu, "Maestro: Replica-aware map scheduling for mapreduce," in CCGrid'12. IEEE, 2012, pp. $435-442$.

[9] Y. Kwon, K. Ren, M. Balazinska, B. Howe, and J. Rolia, "Managing skew in hadoop." IEEE Data Eng. Bull., vol. 36, no. 1, pp. 24-33, 2013.

[10] D. Borthakur et al., "Hdfs architecture guide," 2008.

[11] P. Garraghan, X. Ouyang, R. Yang, D. McKee, and J. Xu, "Straggler rootcause and impact analysis for massive-scale virtualized cloud datacenters," IEEE Transactions on Services Computing, 2016.

[12] L.-C. Canon and E. Jeannot, "A comparison of robustness metrics for scheduling dags on heterogeneous systems," in Cluster. IEEE, 2007, pp. $558-567$.

[13] _ _ "Evaluation and optimization of the robustness of dag schedules in heterogeneous environments," IEEE TPDS, vol. 21, no. 4, 2010.

[14] L. Stanisic, S. Thibault, A. Legrand, B. Videau, and J.-F. Méhaut, "Faithful performance prediction of a dynamic task-based runtime system for heterogeneous multi-core architectures," CCPE, vol. 27, no. 16, pp. 40754090, 2015.

[15] L. Stanisic, "A reproducible research methodology for designing and conducting faithful simulations of dynamic hpc applications," Ph.D. dissertation, Université de Grenoble, 2015.

[16] E. Agullo, L. Giraud, and S. Nakov, "Task-based sparse hybrid linear solver for distributed memory heterogeneous architectures," in Europar, 2016. [Online]. Available: https://project.inria.fr/chameleon 
[17] O. Beaumont, L. Eyraud-Dubois, and Y. Gao, "Influence of Tasks Duration Variability on Task-Based Runtime Schedulers," 2018. [Online]. Available: https://hal.inria.fr/hal-01716489

[18] H. Casanova, A. Giersch, A. Legrand, M. Quinson, and F. Suter, "Versatile, scalable, and accurate simulation of distributed applications and platforms," Journal of Parallel and Distributed Computing, vol. 74, no. 10, pp. 2899-2917, 2014.

[19] D. P. d. Silva, W. Cirne, and F. V. Brasileiro, "Trading cycles for information: Using replication to schedule bag-of-tasks applications on computational grids," in Euro-Par, 2003, pp. 169-180.

[20] J. Dean and G. Sanjay, "Mapreduce: Simplified data processing on large clusters," Communications of the ACM, vol. 51, no. 1, pp. 107-113, 2008.

[21] P. Garraghan, X. Ouyang, R. Yang, D. McKee, and J. Xu, "Straggler rootcause and impact analysis for massive-scale virtualized cloud datacenters," IEEE Transactions on Services Computing, 2016.

[22] T. Phan, A. G., I. S., and R. P., "A new framework for evaluating straggler detection mechanisms in mapreduce," in Proceedings of ICIP 2017, 2017.

[23] M. Zaharia, A. Konwinski, A. D. Joseph, R. Katz, and I. Stoica, "Improving mapreduce performance in heterogeneous environments," in USENIX conference on Operating systems design and implementation. ACM, 2008, pp. 29-42.

[24] Z. Zhu, G. Zhang, M. Li, and X. Liu, "Evolutionary multi-objective workflow scheduling in cloud," IEEE TPDS, vol. 27, no. 5, 2016.

[25] Q. Chen, C. Liu, and Z. Xiao, "Improving mapreduce performance using smart speculative execution strategy," IEEE Transactions on Computers, vol. 63 , no. 4, pp. 954-967, 2014.

[26] Z. Qiu and J. F. Pérez, "Evaluating replication for parallel jobs: An efficient approach," IEEE TPDS, vol. 27, no. 8, pp. 2288-2302, 2016.

[27] D. Wang, G. Joshi, and G. Wornell, "Using straggler replication to reduce latency in large-scale parallel computing," ACM SIGMETRICS Performance Evaluation Review, vol. 4, no. 3, pp. 7-11, 2015.

[28] — - "Efficient task replication for fast response times in parallel computation," ACM SIGMETRICS Performance Evaluation Review, 2014.

[29] L.-C. Canon, E. Jeannot, R. Sakellariou, and W. Zheng, "Comparative evaluation of the robustness of dag scheduling heuristics," in Grid Computing. Springer, 2008, pp. 73-84. 
[30] E. Agullo, O. Beaumont, L. Eyraud-Dubois, and S. Kumar, "Are Static Schedules so Bad? A Case Study on Cholesky Factorization," in 2016 IEEE International Parallel and Distributed Processing Symposium, IPDPS 2016, Chicago, IL, USA, May 23-27, 2016, 2016, pp. 1021-1030. 